\title{
La didactique des langues - cultures à la croisée des méthodes
}

\section{Françoise Demougin}

\section{(2) OpenEdition}

1 Journals

Édition électronique

URL : http://journals.openedition.org/trema/427

DOI : $10.4000 /$ trema.427

ISSN : 2107-0997

Éditeur

Faculté d'Éducation de l'université de Montpellier

\section{Édition imprimée}

Date de publication : 1 novembre 2008

Pagination : 101-111

ISSN : 1167-315X

\section{Référence électronique}

Françoise Demougin, «La didactique des langues - cultures à la croisée des méthodes », Tréma [En ligne], 30 | 2008, mis en ligne le 01 novembre 2010, consulté le 19 avril 2019. URL : http:// journals.openedition.org/trema/427 ; DOI : 10.4000/trema.427

Ce document a été généré automatiquement le 19 avril 2019.

Trema 


\title{
La didactique des langues - cultures à la croisée des méthodes
}

\author{
Françoise Demougin
}

$1 \quad$ L'installation depuis plusieurs années du concept de langue - culture en didactique des langues entraîne de fait dans l'enseignement - apprentissage d'une langue un passage à l'inter - et même à l'intra - culturel, un lien entre items idiomatiques et items culturels, une conjonction d'apprentissages linguistiques, instrumentaux et culturels ne va pas de soi, en particulier sur le plan épistémologique, pour le didacticien. Nous nous proposons de préciser les contours et les entours de cette évolution.

\section{L'évolution de l'enseignement - apprentissage des langues}

2 L'enseignement - apprentissage des langues a connu une évolution sensible, dont rend compte le Cadre Européen Commun de Référence (CECR), évolution qui s'inscrit dans un cheminement historique voyant se succéder des méthodes fondées sur des conceptions différentes de la langue 2. Le tableau ci - dessous rend compte des trois méthodes successivement utilisées :

\begin{tabular}{|c||c||c|}
\hline APPROCHES & $\begin{array}{c}\text { CONCEPTION DE LA } \\
\text { LANGUE }\end{array}$ & COMPÉTENCES VISÉES \\
\hline Méthode audiovisuelle & $\begin{array}{c}\text { ensemble de } \\
\text { structures } \\
\text { permettant un } \\
\text { apprentissage par } \\
\text { répétition }\end{array}$ & $\begin{array}{c}\text { Linguistiques « pré-communicatives « à travers } \\
\text { des activités de compréhension et de production } \\
\text { orales guidées (l'écrit étant un « oral scripturé « } \\
\text { comme le souligne Danielle BAILLY- 1997) }\end{array}$ \\
\hline
\end{tabular}




\begin{tabular}{|c|c|c|}
\hline $\begin{array}{l}\text { Approche } \\
\text { communicative et } \\
\text { cognitive }\end{array}$ & $\begin{array}{c}\text { = moyen de } \\
\text { communication } \\
\text { authentique, outil à } \\
\text { maîtriser }\end{array}$ & $\begin{array}{l}\text { Linguistiques et fonctionnelles à travers des } \\
\text { activités langagières de réception et de } \\
\text { production, à l'oral et à l'écrit. De réflexion sur } \\
\text { la langue, l'apprentissage, la culture à travers } \\
\text { des activités de conscientisation. }\end{array}$ \\
\hline $\begin{array}{l}\text { Approche actionnelle } \\
\text { européenne (Ce que } \\
\text { Christian PUREN(2002) } \\
\text { appellerait « une } \\
\text { perspective co- } \\
\text { actionnelle co- } \\
\text { culturelle») }\end{array}$ & $\begin{array}{c}\text { = moyen } \\
\text { d'interaction } \\
\text { sociale }\end{array}$ & $\begin{array}{l}\text { Linguistiques (lexicales, grammaticales, } \\
\text { sémantiques, phonologiques orthographiques) } \\
\text { Socio - linguistiques (marqueurs linguistiques } \\
\text { de relations sociales, conventions de politesse, } \\
\text { expressions de sagesse populaire, différences de } \\
\text { registres, dialectes et accents) Pragmatiques } \\
\text { (compétence discursive, compétence } \\
\text { fonctionnelle) à travers des activités langagières } \\
\text { de réception, production, interaction, médiation } \\
\text { (à l'oral et à l'écrit). }\end{array}$ \\
\hline
\end{tabular}

On le voit, l'objet enseigné se complexifie avec le temps, proportionnellement à la complexification de la notion de communication. Il est en effet plus difficile d'enseigner une compétence de communication par le biais d'activités requérant l'utilisation de la langue 2 dans des situations réelles et interactives, qu'une compétence "pré communicative ", selon l'expression de S. BOLTON (1991), visant une maitrise d'éléments isolés lexicaux ou syntaxiques.

4 La notion même de compétence a par ailleurs évolué. En linguistique le terme réfère à une capacité sous - jacente, opposée à la performance, qui est La manifestation langagière de la compétence. Dans l'approche communicative ${ }^{1}$, la compétence est définie comme la capacité à utiliser un langage approprié dans des situations et des dispositifs de communication variés. Depuis le milieu des années 90 , la perspective actionnelle est ainsi privilégiée, qui considère les apprenants d'une langue comme des acteurs sociaux, l'interaction comme une façon d'agir avec l'autre'2

Dans le CECR, la compétence de communication langagière est ainsi définie comme la capacité à réaliser une activité langagière pour réaliser une tâche sociale. Le CECR établit aussi une distinction entre l'évaluation du savoir (ou du niveau), qui est « l'évaluation de l'atteinte d'objectifs spécifiques ", " centrée sur le cours », correspondant à " une vue de l'intérieur», et l'évaluation de la capacité (mise en oeuvre de la compétence ou performance), c'est - à - dire « de ce que l'on peut faire ou de ce que l'on sait en rapport avec son application au monde réel ». L'évaluation de la capacité correspond «à une vue de l'extérieur ». On aura compris que idéalement, dans le contexte d'enseignement et d'apprentissage de l'approche communicative, l'écart entre l'évaluation du savoir et l'évaluation de la capacité devrait être infime.

\section{Une prise en compte didactique difficile}

6 Alors que les méthodes didactiques d'enseignement des langues étrangères - du Français en particulier - dites communicatives, dont nous disposons aujourd'hui, sont le résultat de longs travaux de recherche dans plusieurs pays, les méthodes d'enseignement de la culture étrangère (entendue au double sens d'objet et de rapport - CHARLOT 97) sont 
encore en cours de construction. Plusieurs chercheurs ont déjà souligné ${ }^{3}$ qu'en dépit d'un large éventail de publications, il manque à l'étude de la culture un axe précis, celui des recherches théoriques, avec le regret notamment que a rare recherche empirique existante a été effectuée sur une petite échelle et vient souvent de la pratique individuelle des enseignants, sans considération théorique, et s'intéresse plus aux résultats qu'aux processus d'enseignement et d'apprentissage.

7 L'approche communicative a mis ainsi en lumière la complexité de l'apprentissage d'une langue, a fortiori étrangère, sans vraiment donner des réponses didactiques à cette complexité. D'où la tentation dans les pratiques enseignantes de répondre « au coup par coup » à ce qui ressemble à une gageure didactique. Ch. PUREN en 1994, expliquait déjà que la «logique de la variation» plaçait l'enseignant obligatoirement dans «un paradigme de la complexification» (celui précisément de l'éclectisme) ...; il citait, entre autres, la définition que donne S. MOIRAND du terme "approche », préféré au terme «méthodologie " par les tenants de l'approche communicative :

"l'approche n'est pas une étude: c'est un des moyens employés qui permet l'étude d'un sujet considéré comme rebelle à l'analyse, une "forteresse imprenable " [cela implique] 1 / que l'objet à étudier n'est pas a priori connaissable 2 / que la méthode à employer n'est pas a priori définie ».

Dans cette perspective aucun modèle théorique préétabli ne peut apporter de réponse satisfaisante, et donc « il ne s'agit plus d'appliquer le plus systématiquement possible tel ou tel modèle a priori, mais au contraire d'adapter avec le plus de souplesse possible des modèles les plus variés possibles à des paramètres situationnels [...] considérés comme des contraintes, des données ou des construits ${ }^{5}$ ». Cela oblige, écrivait encore PUREN, à procéder aux "nécessaires remaniements épistémologiques de la didactique ", en privilégiant « l'incertitude et le dialogique ${ }^{6} »$. Il s'appuyait pour cela sur les travaux qu'E. MORIN a consacrés à la pensée complexe, qu'il décrit comme " animée par une tension permanente entre l'aspiration à un savoir non parcellaire, non cloisonné, non réducteur, et la reconnaissance de l'inachèvement et de l'incomplétude de toute connaissance ${ }^{7}$ ».

9 On ne peut qu'être d'accord avec ce constat. Mais l'éclectisme est- il la seule réponse possible, la plus appropriée ? Il nous semble nécessaire au contraire d'aller au - delà d'une recherche empirique, liée à des résultats forcément ponctuels, obtenus par des

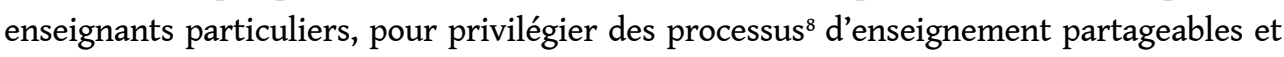
formalisables. C'est à ces processus que nous allons maintenant nous intéresser.

\section{La réponse de la didactique contextualisée}

\section{1 La prise en compte de la complexité}

10 La prise en compte de la complexité paraît devoir être davantage conjonctive que disjonctive et entraîner de ce fait: 1 / une place importante réservée à la pratique raisonnée de la langue. 2 / une recherche de transversalité. 3 / une réflexion sur la globalité de l'activité de langage (impliquant la prise de conscience que l'apprentissage d'une langue forme un tout cohérent, qu'il y a des liens entre lexique, grammaire, phonologie et culture notamment). La gestion de la complexité n'impose pas, à l'instar de la pédagogie par objectifs, une segmentation systématique et un traitement dissocié des difficultés, mais il est possible, comme le préconise le même MORIN (1997), de procéder à «une réforme, non pas programmatique mais paradigmatique qui concerne notre 
aptitude à organiser la connaissance ", et qui permettrait de privilégier l'articulation entre les diverses composantes plutôt que leur disjonction. On est bien davantage dans une logique de la connexité, dans la perspective de ce que nous avons appelé une anthropologie de contextualité, qui oblige à la prise en compte d'une compétence culturante (renvoyant à des savoirs questionnés par l'expérience personnelle de la connaissance, DEMOUGIN 2006) aux côtés d'une compétence culturelle (qui, elle, renvoie à une connaissance questionnée par l'état des sciences contemporaines).

\section{III.2 La prise en compte du cadre}

11 Quel est le cadre de ces processus d'enseignement que nous évoquons? L'enseignement / apprentissage d'une langue - culture répond à une injonction contradictoire de mobilisation d'identités multiples et de confrontation à une altérité résistante, la langue de l'autre ${ }^{9}$. Apprendre une langue (étrangère ou seconde) va de fait consister pour l'apprenant à élaborer, à partir de son interaction avec le matériau linguistique et langagier contextualisé qu'il rencontre, un nouveau système explicatif, en corrélation interne avec le système de sa langue maternelle (ou de l'usage ordinaire de sa langue), et en corrélation externe avec les systèmes explicatifs de ses interlocuteurs. S'il est vrai que le sujet reconstruit l'objet selon un processus qui lui est propre, il n'en reste pas moins que l'objet pré - existe avec sa cohérence interne, dont il est nécessaire pour chacun de se rapprocher le plus possible. Ce double mouvement de construction personnelle et de contrôle par le contexte prend un intérêt tout particulier dans l'apprentissage scolaire. D'autant que la langue - culture proposée par l'école, qu'elle soit au demeurant seconde, étrangère ou maternelle, reste foncièrement langue - culture de l'autre, qui engendre deux risques complémentaires : celui de la ressentir comme extériorité à soi et de se replier sur la position scolaire passive d'un apprentissage qui sert à quelque chose, celui aussi de la ressentir comme aspiration à être au monde, dans la confusion de tous les genres et la relativité de tous les savoirs. Ces risques assumés, et théoriquement dépassés par la dialectique du même et de l'autre fondatrice du sujet, restent malgré tout résistants.

\section{3 Un continuum des contextes}

Le travail doit se faire autour d'un continuum entre langue objet, langue référence et langue moyen d'enseignement - apprentissage, par la prise en compte des contextes institutionnels, sociolinguistiques, culturels, par la prise en compte des codes linguistiques, éducatifs, didactiques et culturels. On peut faire l'hypothèse que ces contextes produisent des effets notables sur les modalités d'enseignement - appropriation des langues et vice - versa, qu'il n'y a par conséquent pas de didactique universelle des langues mais plutôt des didactiques contextualisées et historicisées. Qu'est-ce que cela veut implique? :

- que toute construction de connaissances disciplinaires dans un système éducatif passe, à des degrés et sous des modes variés, par un travail linguistique qui contribue à l'abstraction, à la conceptualisation, à la mise en relation de concepts, plus généralement à l'élaboration cognitive d'un domaine (CASTELLOTI et MOORE : 97, VIGNER 2001). Les connaissances disciplinaires ne sont pas seulement d'ordre déclaratif et de représentation propositionnelle, elles sont aussi d'ordre procédural et de représentation actionnelle, 
touchant à des séquences relevant de savoir - faire plus que de savoirs, au sens habituel de ce dernier terme. Les activités qui constituent un domaine de connaissance mobilisent des opérations et pas seulement des notions (COSTE 2005). Ainsi une analyse des manuels en vigueur en France et des pratiques, prescrites autant qu'effectives, dans les classes du secondaire et du primaire permet de relier les consignes d'activités à cinq opérations intellectuelles privilégiées qui constituent l'ossature de l'activité scolaire, quand ce n'est pas la totalité de cette activité. Il est en effet demandé à l'élève essentiellement de repérer, de classer, de répéter, d'induire, de comparer ${ }^{10}$.

- qu'existent donc des codes didactiques, éducatifs, et non seulement linguistiques, différents. La langue - source constitue un filtre de la connaissance, ce qui ne veut pas dire un obstacle, par les pratiques culturelles d'apprentissage qu'elle véhicule. Ces pratiques culturelles réunissent les différents champs disciplinaires (par exemple par le recours à certains outils transversaux, comme le tableau à double entrée très utilisé en France) ou les différencient (le commentaire littéraire a peu de choses à voir avec le commentaire historien par exemple). Ces codes didactiques et éducatifs se développent donc de manière à la fois interne et externe à la langue - culture.

- que le détour - recours à une langue - cible, à une autre langue que la sienne, peut être une source d'approfondissement pour la maîtrise langagière. Ainsi la comparaison du système français avec celui d'autres langues permet à la fois : la prise en compte de la diversité linguistique de plus en plus grande des classes, la mise en place de démarches réflexives qui tiennent compte du « déjà-là » de l'élève, une attitude active de compréhension et d'appréhension de sa propre langue. Lorsque nous cherchons à comprendre un texte dans une langue « étrangère ", c'est-à-dire que nous ne connaissons pas, mais qui est proche (ceci ne signifie pas nécessairement de la même famille) d'une autre langue déjà connue, maternelle ou étrangère, nous mettons en relation assez facilement les segments « transparents » entre les langues (pas seulement lexicaux, syntaxiques et phonologiques aussi). Mieux, nous pouvons comprendre aussi, très régulièrement, des segments qui nous semblaient peu transparents au premier abord, ou qui peuvent demeurer non transparents, même s'ils ont été devinés. Cette extraction de la connaissance a été d'autant mieux vérifiée et rendue performante quand on a décidé d'aborder plusieurs langues de la même famille dans la même séance. Il est plus aisé de mettre en place un réseau d'acquisition de la connaissance et de mémorisation de cette connaissance entre quatre langues plutôt qu'entre seulement deux langues. En abordant simultanément plusieurs langues de la même famille, on ne se contente pas de poser des équivalences ou des absences d'équivalence entre la langue visée et la langue maternelle. On met en place un réseau de similitudes et de dissimilitudes, et la compréhension des langues est acquise dans le cadre de ce réseau. Dès ce moment, les élèves commencent un voyage linguistique qui les mène aussi chez eux. Chacun connaît ainsi l'exemple de l'Histoire où le même événement sera nommé différemment dans deux langues : le « Débarquement » français du 6 juin 1944 devient l'» Invasion » en allemand, et l'on voit comment ces deux termes n'entrent pas dans les mêmes réseaux sémantiques dans l'une et l'autre langue, n'y présentent pas les mêmes connotations. Mobiliser les deux langues c'est alors enrichir l'une et l'autre à travers l'approfondissement des représentations que chacune propose de ces notions historiques, et enrichir le champ historique aussi. C'est provoquer encore une fois une décentration bénéfique à la maîtrise de la langue ${ }^{11}$.

- que l'évaluation ne peut faire abstraction de cette culturalisation des savoirs : en définissant le locuteur comme un acteur social susceptible d'effectuer des tâches requérant à la fois des compétences générales et des compétences langagières, en prenant davantage en compte ce 
qui ressort de la complexité identitaire ${ }^{12}$, en procédant notamment à une inversion méthodologique qui mènerait de la culture de référence à la culture - cible. Avec un double avantage : ancrer la parole de l'apprenant et éviter un déséquilibre excessif entre les interactants (où l'un serait en position dominante d'expert de la langue et de la culture par rapport à l'autre, de par son savoir, son savoir - faire et son savoir dire). Prendre en compte de ce fait le discours, et déplacer le centre d'intérêt de l'objet langue vers le couple locuteur - récepteur, vers la contextualisation énonciative. En considérant de ce fait le déjà - là comme un moteur et non comme un frein de la conscience linguistique : la prise de conscience de son multilinguisme constitue pour l'apprenant un espace cognitif réel. En permettant à l'apprenant d'expliciter son expérience de l'écart, du décalage, voire de la rupture. Et pour cela en évitant les supports ou les formes de questionnement trop normés. En permettant à la langue - cible de se déployer dans un usage personnellement assumé au cœur d'une " posture désirante ${ }^{13}$ ». On voit assez ici le rôle que pourraient jouer des supports comme l'image ou le texte littéraire, dans une double dynamique : a / l'inscription d'une identité culturelle en évolution dans un processus d'acquisition, b / l'inscription par le locuteur de traces de son être dans la communication. En dépassant en définitive une logique de type additionnel pour aller vers une approche didactique intégrée, qui se heurte pour le moment à un découpage disciplinaire.

13 Les outils européens semblent déjà privilégier une estimation positive, valorisante tout en restant objective, en fonction de critères de réussite précis selon une nomenclature d'actes sociaux à accomplir dans des contextes variés. Pour se figurer la révolution mentale que le CECR nous demande d'effectuer, on peut utiliser l'image dans le cas de notre tradition d'un palier situé en hauteur sans marches pour l'atteindre - la norme étant la langue du natif, et dans le cas des outils européens, un escalier menant progressivement au même palier mais où l'on est en droit de stationner confortablement à chacune des marches: A1, A2, B1, B2, C1, C2, car la "norme » n'est plus une mais multiple. Grâce à cette image, on peut aussi concevoir différemment les démarches d'enseignement - apprentissage en amont: dans un cas, enseigner c'est vouloir faire atteindre un niveau d'excellence, dans l'autre, c'est faire passer d'un niveau à un autre qui lui est supérieur. Dans le premier cas, l'évaluation a de grandes chances de s'effectuer sur la base de la frustration, dans le second, sur celle du contentement. En fait c'est la notion même de réussite qui est en jeu ici avec l'idée, dans le premier cas, que finalement tout le monde échoue sauf les majors des grandes écoles ou comme le disent BLAIS, GAUCHET et OTTAVI (2003) que « l'étudiant des Mines a raté Polytechnique et le certifié l'agrégation ». Pour résumer la perspective du CECR : évaluer c'est « donner de la valeur » / réussir c'est réussir une tâche / enseigner c'est faire passer d'un niveau au niveau immédiatement supérieur. Toutes ces avancées, car c'en sont, sont liées, on le voit, principalement et peut-être essentiellement, à la prise en compte de la dimension sociale et culturelle de la langue objet d'enseignement et d'apprentissage. Tendance illustrée aujourd'hui par les recherches conduites en didactique sur le concept d'appropriation.

\section{Le concept d'appropriation et les pratiques culturelles}

Qu'est-ce en effet qu'apprendre une langue (fût-elle étrangère, seconde ou maternelle) sinon se l'approprier en tant qu'expression de soi, c'est-à-dire de son rapport à l'Autre, au sein d'une communauté qui, quelle qu'elle soit, « exige » en quelque sorte de la « solidarité 
»? Les pratiques culturelles, au sens large, semblent à ce titre précieuses, qui sont une entrée possible dans l'univers de l'autre en même temps qu'un lieu de construction de soi. La littérature, par exemple, apparaît ici moins comme ce qui unit HOMÈRE à MALLARMÉ que comme ce qui fait du lecteur un locuteur pris dans la double tension de soi et de l'autre. Si l'horizon de l'Encyclopédie Universalis reste vierge de tout article « littérature ", c'est précisément parce que la littérature est un usage particulier du langage, une certaine manière de faire fonctionner une langue et d'en expliciter les possibilités. Il n'y a là aucune restriction, bien au contraire, une expansion infinie qui donne tout son prix à la littérature et l'associe à la science du langage. D'autant que le texte littéraire ${ }^{14}$, loin de n'être qu'un bluff visant à une surprise éphémère, peut mener lors d'une seconde lecture à une réflexion sur le statut illocutif et la portée référentielle de la fiction. L'immersion fictionnelle, comme l'immersion linguistique et culturelle, favorisent la «sculpture de soi » et des autres, la ferveur du dedans et la " ferveur du dehors ${ }^{15}$ ». Grandir dans une culture, dans une langue c'est en accepter en principe toutes ses limites. Cependant il se pourrait bien que la littérature n'existe que pour mieux nous obliger à transgresser ces limites. Où l'on voit l'importance de la démarche littéraire ${ }^{16}$, que sa perspective heuristique place au cœur de la dimension inter- et intraculturelle ... L'expérience littéraire fonctionne en effet comme une expérience à la fois collective et individuelle, qui engage des conséquences langagières et identitaires dont l'importance n'apparaît pas forcément immédiatement à celui qui la vit. Mais cette expérience marque durablement le locuteur, il n'en faut pas douter.

Ce faisant, on fait apparaître l'importance des différents contextes: scolaire, social, symbolique, dans la construction identitaire de l'élève et le danger de laisser s'installer une certaine forme de "misère symbolique ${ }^{17}$ " induisant à terme l'exhibition des particularismes d'une part et le formatage intellectuel d'autre part. Tout projet didactique alors devrait tendre à construire un véritable « nous " par l'usage de pratiques langagières tournées vers la négociation et le débat, à permettre une prise de parole à la fois collective et individuelle, à rétablir un circuit du désir, toutes choses tournées vers la reconquête d'un sens esthétique et axiologique, d'un savoir doublé d'un non - savoir toujours en devenir, vers la reconquête d'une parole. On mesure dans cette perspective à la fois la priorité à accorder à l'oralité et à la discussion, et l'importance du choix des supports. Le support culturel, et particulièrement littéraire, y retrouve sa fonction sociale ${ }^{18}$, car la compréhension du livre est forcément disputée. Le texte lu n'y sert pas à évaluer, à sanctionner une compétence de lecture, il apprend à penser, à se construire comme sujet dans l'espace classe. Cela n'abolit pas toute violence, mais cela permet au moins à la parole de (ré) apparaître comme lieu d'échange et d'écoute. En dernière analyse c'est bien la question de l'enseignement de la culture à l'école qui est soulevée. Enseigner la culture, c'est moins se frotter à une culture littéraire française hiérarchisée que se construire, dans la langue qu'on apprend et qui est forcément, même maternelle, celle de l'autre, une nouvelle médiation au monde. Le recentrage sur une dimension culturelle et subjective opère ainsi une mise en tension des pôles linguistique et anthropologique. Ce qui implique une action non tant sur le savoir lui-même que sur le rapport au savoir.

16 L'enjeu est bien de prendre en compte à la fois les fonctionnements sociaux de la langue, objet d'enseignement et d'apprentissage, et ses fonctionnements plus globalement culturels, notamment dans leurs liens avec les imaginaires collectifs. En replaçant l'apprentissage d'une langue dans la perspective d'un désir d'expression par le locuteur de ce qu'il sent, ce qu'il sait, ce qu'il croit savoir : le désir de dire allant de pair avec le 
désir d'apprendre à dire. Et ce n'est pas là seulement reprendre les thèses constructivistes mais bien travailler autrement l'inter - et l'intra - culturel, en phase avec l'intérité dont parle le sociologue J. DEMORON, souvent cité par nos collègues de didactique des langues. L'enjeu, précisément, en est la reconfiguration de la langue par le locuteur lui-même, le locuteur réel, empirique dirait U. ECO. L'enjeu en est de réinscrire le geste de dire dans l'acte d'apprendre, de donner son espace à la langue ordinaire en continuum avec la langue apprise, de promouvoir un " trajet de langue » avec les creux de ce qui ne " parle » guère ou qui parle mal et les bosses de ce qui excède la parole. De retrouver ce locuteur indocile, mais non fautif, qui construit son identité langagière par racines et par rhizomes, au cours et au cœur d'un apprentissage délinéarisé. D'éviter que ne se creuse entre le Sa et le Sé un divorce de plus en plus grand : l'enjeu des apprentissages culturels est bien de re-matérialiser la parole, de lui redonner sa dimension fondatrice. De laisser apparaitre, voire de provoquer, une parole trébuchante, c'est-à-dire à la fois hésitante, si l'on prend le sens commun du terme, mais aussi «correcte au poids " au sens où l'on parle d'une monnaie trébuchante. Il est bien question d'échange et de vie sociale à ce niveau.

Le titre de cet article : "la didactique des langues - cultures à la croisée des méthodes ", se référait explicitement au titre de l'ouvrage de Ch. PUREN. C'est cette croisée - là qui nous intéresse et fait apparaître de plus en plus la didactique comme une science de la convergence, un lieu crucial, carrefour de complexités multiples, celles de l'élève, celles du maître, celles des processus cognitifs sollicités, comme un lieu par conséquent d'inventivité aussi, où la construction de soi va de pair avec la réduction de l'écart entre culture normative prescrite et culture descriptive construite, entre l'ensemble des œuvres construites par l'humanité pour se comprendre elle-même dans le monde et l'ensemble des modes de vie, de comportements et des croyances d'un groupe social, va de pair avec une didactique ouverte à d'autres disciplines appartenant aux sciences humaines et sociales comme l'anthropologie, la psychologie, les sciences cognitives, l'histoire ou la sociologie, avec une didactique intégrée qui dépasse une logique de type additionnel pour aller vers une logique de type évolutif - intégratif. Qui est encore à fabriquer.

\section{BIBLIOGRAPHIE}

BLAIS, M-Cl., GAUCHET, M., OTTAVI, D. (2003). Pour une philosophie politique de l'éducation, Paris, Hachette Pluriel Référence.

BOLTON, S. (1991). Évaluation de la compétence communicative en langue étrangère, Paris, Hatier, Didier, collection LAL.

CASTELLOTTI, V. et MOORE, D. (1999). «Alternance des langues et construction des savoirs », in Cahiers $d u$ français contemporain, ENS Fontenay - Saint-Cloud, Paris, CREDIF.

CHARLOT, B. (1997). Du Rapport au savoir. Éléments pour une théorie, Paris, Anthropos. 
COSTE, D. (2005). « Construire des savoirs en plusieurs langues. Les enjeux disciplinaires de l'enseignement bilingue », Santiago de Compostelle.

DEMORGON, J. (2005). Critique de l'interculturel, L'horizon de la sociologie, Economica - Anthropos.

DEMOUGIN, F. (2006). Apprentissages culturels et enseignement de la langue, mémoire de soutenance de HDR, Université de Nice - Sophia Antipolis, 2 décembre 2006.

DEMOUGIN, F. (coord.) (2006). Lire dans la langue de l'autre. Montpellier, Presses de l'Université Montpellier III - Paul Valéry.

DUMONT, F. (1968) Le lieu de l'homme. La culture comme distance et mémoire. Montréal, Éditions HMH (collection Constantes, 14), Bibliothèque québécoise, 1994.

ECO, U. (1985). Lector in fabula, le rôle du lecteur ou la coopération interprétative dans les textes narratifs , trad. française Paris, Grasset et Fasquelle.

MORIN, E. (1982). Science avec conscience, Paris, Fayard.

PETIT, M. (2002). Éloge de la lecture. La construction de soi, Paris, Belin.

PUREN, Ch. La didactique des langues étrangères à la croisée des méthodes : essai sur l'éclectisme, Didier - Crédif, École Nationale Supérieure de Fontenay / Saint-Cloud. « Le CECR et la réflexion méthodologique en didactique des langues - cultures : un chantier à reprendre ».

STIEGLER, B. (2004). De la misère symbolique (1- L'époque hyperindustrielle), Paris, Galilée.

VIGNER, G. (2001). Enseigner le français comme langue seconde, Paris, CLE International.

\section{NOTES}

1. Rappelons brièvement les différentes compétences : la compétence lexicale (mise en mémoire de représentations lexicales, acquisition de procédés de contournement des lacunes lexicales inévitables en situation de production - écrite ou orale - ou de réception), la compétence linguistique (intériorisation des règles de communication naturelles, compétences grammaticale et phonétique, toute la surface linguistique qui reflète l'éthos communicatif de chaque culture), la compétence socio - culturelle (savoirs inhérents à la culture, rituels d'interaction, normes d'interprétation), la compétence pragmatique (interaction linguistique entre les participants, codes sociaux internes).

2. L'interculturel visait à éviter de tomber dans l'assimilation à l'ancienne d'une part et de l'autre dans le communautarisme, à rétablir l'idée d'échanges que ne mettait pas suffisamment en avant le multiculturel, le co-culturel vise à mettre en avant une dimension pragmatique féconde tendant pourtant à une co-neutralisation des idées et des oppositions qui peut poser questions. Ces réponses didactiques apportées à l'exigence d'altérité s'inscrivent, et c'est cela en réalité qui pose questions, dans le cadre général d'un relativisme culturel pensé de plus en plus comme une fin des cultures et donc des conflits. Or, pas plus qu'il ne peut y avoir de fin de l'histoire pour l'historien il ne peut y avoir de fin des cultures pour le didacticien des langues. C'est un des apports justement du transculturel, dernière dérivation apparue pour le terme "culture », que de permettre de mettre en avant l'idée de mouvement, de progression au lieu de celle de terme : la culture ne saurait effectivement être résumée à un acquis de connaissances, à un empilement de savoirs. Si en effet l'existence de valeurs universalisables, mises en lumière par le transculturel, est claire, ces dernières ne peuvent pour autant définir la culture comme un agrégat de valeurs capitalisables. L'idée de culture renvoie bien davantage à une posture éthique, culturante plus que culturelle, dans la mesure où elle apparaît comme un questionnement par 
l'expérience personnelle de la connaissance, des savoirs, sans que ceux-ci soient occultés. Ce questionnement prend en compte la mise en tension de différentes pratiques culturelles, collectives et individuelles, et suppose une vision dynamique de l'apprentissage qui ne se limite pas à un discours théorique, général et décontextualisé. Il évite la bi - focalisation sur une culture première, acquise, qui s'opposerait à une culture seconde, en voie d'acquisition, il évite une résolution au bénéfice d'une forme culturelle normée qui fasse l'impasse de la saisie du savoir expérientiel de l'apprenant.

3. M. BYRAM en particulier.

4. Dans La didactique des langues étrangères à la croisée des méthodes : essai sur l'éclectisme, Didier Crédif, 1994, Ecole Nationale Supérieure de Fontenay / Saint-Cloud, p. 62.

5. Op. cit. p. 165.

6. Ibid., p. 123.

7. Cité par PUREN.

8. Nous ne parlons pas ici de gestes professionnels mais bien de processus d'enseignement.

9. À ce sujet voir Lire dans la langue de l'autre (dir. F. DEMOUGIN), 2006, Presses de Montpellier III.

10. Relevé mis au point par notre équipe engagée dans le projet INRP : « entré dans l'écriture : points de résistance à l'apprentissage de la langue / langue, culture, interculturalité ».

11. Un projet comme Euromania fonctionne sur ce principe structurel, tout comme le rejoignent certaines approches grammaticales de la langue fondée sur une didactique comparée des langues.

12. La prise en compte de l'apprenant qui apparaît ici au centre du système d'apprentissage est confortée par la popularisation des théories constructivistes de PIAGEt et VYGOTSKY, dans lesquelles on part du principe que l'enseignant de FLE est là pour aider l'apprenant à construire son apprentissage (par l'appropriation des savoirs mais aussi des savoir - faire), en l'aidant à découvrir ses stratégies et ses styles d'apprentissage (les savoir - apprendre) pour en définitive l'aider à se construire en tant que personne (savoir - être).

13. Nous entendons par ce terme la mise en place chez l'élève d'un "désir de parole", inséparable pour nous de la recherche d'une habileté langagière. On se rappelle le propos de FREUD : «Apprendre c'est investir du désir dans un objet de savoir ».

14. dont J. SEARLE parlant plus précisément de la fiction rappelle qu'il est une feintise réglée par des conventions pragmatiques, par conséquent extérieures aux énoncés et tenant au contexte culturel qui admet cette sorte de jeu de langage qu'est la feintise littéraire.

15. Ce dernier terme est emprunté à l'écrivain P. BERGOUNIOUX, cité par M. PETIT dans son Éloge de la lecture, pour qui il existe d'autres choses que celles qui nous entourent, liées aux contrées lointaines, qu'apporte la lecture de textes littéraires.

16. Démarche littéraire qui n'a rien à voir avec l'activité littéraire telle qu'elle peut être pratiquée dans un cours "normé » de littérature, où le discours sur le texte est programmé à l'avance par un savoir sur le texte littéraire au demeurant tout à fait respectable.

17. On reconnaîtra là une expression du philosophe $B$. STIEGLER. La misère symbolique correspond pour lui à la perte d'individuation qui résulte de la perte de participation à la production des symboles. L'effondrement symbolique qu'il décèle dans nos sociétés hyper industrielles mène à une décomposition préoccupante du lien social.

18. Souvent oubliée au profit d'une fonction « distinctive » mise en avant par un système scolaire élitaire. 


\section{RÉSUMÉS}

L'installation du concept de langue - culture en didactique des langues pose le problème de la prise en compte de la complexité de l'enseignement - apprentissage d'une langue. Les modèles théoriques existants peinent à donner à l'étude de la culture un axe précis. Des pistes se dégagent, autour de l'étude des processus d'enseignement et d'apprentissage, notamment par le biais du concept d'appropriation et le choix d'une didactique contextualisée, intégrée, qui dépasse une logique de type additionnel.

The complexity of teaching and learning a language is becoming a problem with the development of the concept of «language and culture » in the study of languages. The existing theoretical models hardly give precise guidance as to the studying of a culture. Some ideas are however emerging, from the study of the teaching and the learning process. In particular ideas based on the concept of assimilation and the choice for a method of study which is integrated, in context, and which goes beyond the mere logic of accumulation.

\section{INDEX}

Mots-clés : appropriation, complexité, culture, didactique, langue, méthode

Keywords : assimilation, complexity, culture, language, methodology, study

\section{AUTEUR}

\section{FRANÇOISE DEMOUGIN}

Professeur, Sciences du langage, IUFM de Montpellier - Montpellier 2, Dipralang - Didaxis (EA

739) 